\title{
Die Volksunterrichtspolitik von Bálint Hóman und die Schulgründungen im Komitat Baranya zwischen 1932 und 1942
}

\author{
The Public Education Policy of Bálint Hóman and the School Establishments \\ in Baranya County between 1932 and 1942
}

Bálint Hóman played an important role in the history of Hungarian education. He was Minister of Religion and Education twice between 1932 and 1942, at a time when Europe was experiencing rapid changes in many areas of life, including education. The issue of establishing schools was a significant part of his public education policy in accordance with the Christian-national ideology of the era. In order to evaluate the plans for school building created within the framework of a central education policy, it is necessary to examine their implementation through specific local examples. In my study I am looking for an answer for how the cultural policy of Bálint Hóman appeared in Baranya County: the Baranya County Archives of the Hungarian National Archives provide good examples of the implementation but also the obstacles of establishing schools at a local level.

Keywords: Christian-national education - education policy - school establishments

\section{Einleitung}

Die Modernisierung des Volksunterrichtssystems und die kirchliche Interessendurchsetzung in diesem Prozess in Ungarn nach 1848 im Vergleich zur Rechtsentwicklung in Österreich wird von der Verfasserin dieses Beitrages seit vier Jahren erforscht. ${ }^{1}$ Ein besonders bemerkenswerter Teil der ungarischen Volksunterrichtspolitik der Zwischenkriegszeit bzw. der Jahre des Zweiten Weltkriegs war die Frage der Schulgründungen. Um die im Rahmen der zentralen

\footnotetext{
${ }^{1}$ Weitere Aufsätze in deutscher Sprache: Die Modernisierung des Volksunterrichts in Ungarn - Umsetzung des Gesetzes über den Volksunterricht im Komitat Baranya nach 1868 (Journal on European History of Law 2018); Unterrichtspolitik - kirchliche Interessendurchsetzung in Ungarn nach 1848 (Krakowskie Studia z Historii Panstwa i Prawa 2017).
}

Unterrichtspolitik erstellten Schulgebäudepläne zu bewerten, muss deren Umsetzung anhand konkreter lokaler Beispiele geprüft werden. Im folgenden Aufsatz wird untersucht, wie sich die Kulturpolitik des Ministers für Kultur und Unterricht, Bálint Hóman, im Komitat Baranya ausgewirkt hat: In den Dokumenten der Schulaufsicht von Pécs und Baranya, die im Komitatsarchiv Baranya des Ungarischen Landesarchivs (im Weiteren: MNL BaML) aufbewahrt wurden, kann man gute Beispiele für die lokale Umsetzung und auch die Hindernisse dieser Zielsetzungen finden, die zu der - in der ungarischen historischen und rechtshistorischen Fachliteratur bisher lückenhaften - Aufarbeitung des Themas beitragen können. 


\section{Die Grundlagen der sogenannten nationalen Bildung}

Die Bildungskonzepte im Ungarn der Zwischenkriegszeit waren durch die christlichnationale Denkweise geprägt. Das Wesen der ungarischen kulturellen Überlegenheit war, dass Ungarn seine Position gegenüber den umliegenden Völkern durch Hebung seiner nationalen Kultur stärken konnte. Die Kultusminister István Haller ${ }^{2}$ und József Vass ${ }^{3}$ hatten bereits den Schwerpunkt auf religiöse, patriotische und nationale Erziehung gelegt, doch Kuno Klebelsberg4 entwickelte das Konzept der kulturellen Überlegenheit. Die Unterrichtspolitik von Bálint Hóman basierte auch auf den Ideen seiner Vorgänger, er entwickelte diese Ideen jedoch weiter und versuchte sie in die Praxis umzusetzen. ${ }^{5}$

Nach dem Friedensdiktat von Trianon zogen Lehrer aus den angrenzenden Regionen in das Mutterland; so wurde dieser Beruf (auch) überfüllt. Ein Aspekt für die Auswahl von Lehrern war, ob sie ihrer Verpflichtung zur christlichen Erziehung erfüllt hatten. Dieses Kriterium wurde nicht nur bei der Anklage von konfessionellen Lehrern nach der Räterepublik (sog. Tanácsköztársaság) untersucht, sondern man blickte auch auf frühere Jahre zurück, in denen diese Erwartung selbst noch nicht formuliert worden war. ${ }^{6}$ Religiöse, patriotische, nationale Erziehung wurde Teil der christlich-nationalen Ideologie, ${ }^{7}$ und die Lehrer mussten dies rückwirkend nachweisen. Dementsprechend hatte die Erziehung in der Schule einen höheren Stellenwert als der Unterricht. Die Erwartungen an die Lehrer zeigten sich auch im Wortlaut ihres obligato-

\footnotetext{
${ }^{2}$ Im Amt 24. 11. 1919-16. 12. 1920.

${ }^{3}$ Im Amt 16. 12. 1920-16. 6. 1922.

${ }^{4}$ Im Amt 16. 6. 1922-24. 8. 1931.

${ }^{5} \mathrm{Zu}$ den Reformen von István Haller, József Vass, Kunó Klebelsberg und der ungarischen kulturellen Überlegenheit siehe MANN, Oktatáspolitikusok 15-73. ${ }^{6}$ NAGY, A növekvő állam 249.

${ }^{7}$ Siehe HerGer, Auf dem Weg 69-95.
}

rischen Eides, der insbesondere an dessen Ende zum Ausdruck kommt: „Ich schwöre bei Gott, dass ich Ungarn, der ungarische Verfassung und dem ungarischen Gouverneur fest und unentwegt treu bleibe, ich werde immer die ungarischen Gesetze und Gewohnheiten einhalten, sowie die konstitutionellen Regierungsvorschriften und meine Lehrpflichten zuverlässig, pflichttreu und pünktlich erfüllen. Ich werde die mir anvertraute Jugend im Geiste der Liebe zur Heimat und der religiös-moralischen Bildung erziehen." 8

Die kulturpolitische Auffassung der Zwischenkriegszeit charakterisieren die folgenden Sätze aus dem Artikel vom 15. Januar 1934 des Volkslehrerblatts: „Und wenn die Zukunft der Nation vor uns steht: Dem Kind müssen wir nicht nur das praktische Denken in seiner Seele verankern, sondern auch die idealen Gedanken in ihm erwärmen. Wir müssen es zum moralischen Bewusstsein führen, damit es die feinsten Schattierungen von Gut und Böse beurteilen kann. Und vertiefen wir in seiner Seele die instinktive Kraft der moralischen Belastbarkeit. Glühen wir seine Seele zu hartem Stahl, härten wir seinen Charakter offen und entwickeln wir sein Weltbild in die richtige Richtung. Legen wir großen Wert auf eine zielorientierte nationale Bildung. Jedes Kind soll das Gefühl haben, dass wir den anderen Nationen im Bereich der Kultur gegenüber weit voraus sind, sie sind auch beteiligt und sie können darauf stolz sein, Ungarn zu sein. "9 In diesen Zeilen fühlt man die Leidenschaft für die Ideale der nationalen Bildung und der ungarischen kulturellen Überlegenheit, die die ganze Unterrichtspolitik durchdrangen.

\footnotetext{
${ }^{8}$ Weitere Eide siehe z.B.: MNL BaML VI. 502. 1496/1942, 1614/1942, 1940/1942, 2017/1942, 2090/1942, 2254/1942, 2802/1942, 3753/1942, 3241/1943.

${ }^{9}$ ANONYMUS, A kultúrfölény 41.
} 


\section{Die Kulturpolitik von Bálint Hóman und der Schulbau}

In den dreißiger Jahren vertrat der Kultusminister Bálint Hóman ${ }^{10}$ auch das Ideal der nationalen Bildung. Er spielte eine bestimmende Rolle in der Geschichte des ungarischen Volksunterrichts: Er war zweimal Kultusminister (1. 10. 1932-13.5. 1938 und 16.2. 1939-3. 7. 1942) in Zeiten, als überall in Europa - in vielen Bereichen des Lebens - rasche Veränderungen eingetreten waren, so auch in der Bildung. Die Presse seiner Zeit hielte Hóman für eine besonders positiv zu beurteilende Persönlichkeit. Die folgenden Zeilen im Volkslehrerblatt geben einen Überblick über diese Tatsache: „Wir sind stolz darauf, dass der hochklassige Wissenschaftler, der große Staatsmann, die hervorragende Persönlichkeit unseres wissenschaftlichen, gesellschaftlichen, politischen Lebens nach einer kurzen Unterbrechung wieder unser Führer wurde. $\mathrm{Er}$, der große produktive Minister kennt alle kleinen Punkte unseres Volksunterrichtssystems. Er weiß, wo was gut und richtig ist, wo was schlecht ist, und er, als tiefblickender Wissenschaftler und Kulturpolitiker, findet sofort einen Weg, um zu helfen und zu verbessern. Wir, die ungarischen Lehrer, haben uns schon vielmal geäußert und wir haben immer das Gefühl gehabt, dass die Fäden der Dankbarkeit uns für immer mit Bálint Hóman verbunden haben." ${ }^{11}$ Um ein ganzes Bild von Hóman als Politiker zu bekommen, muss man auch andere Teile seiner Tätigkeit betrachten. Heutzutage gibt es immer noch unterschiedliche Meinungen $^{12}$ zu seiner Person, in erster Linie bezüglich seiner Rolle bei der Schaffung der sogenannten Judengesetze. Nach dem Zweiten Weltkrieg

\footnotetext{
10 *Budapest, 29. 12. 1885, + Vác, 2. 6. 1951; Lebenslauf von Hóman siehe Országgyülési Almanach 196-200.

${ }^{11}$ DrOZDY, Gróf Teleky Pál - Hóman Bálint 150.

${ }^{12}$ Siehe MANN, Oktatáspolitikusok; UjVÁRY, Történeti átértékelés.
}

wurde er vor das Volksgericht gestellt, welches ihn als Kriegsverbrecher verurteilte. ${ }^{13}$ Überraschend für die Nachwelt ist, dass der Grund nicht die Manifestation seiner antisemitischen Ansichten war, sondern Folgendes: Er hatte an der Kabinettssitzung teilgenommen, die den Kriegszustand zwischen Ungarn und der Sowjetunion billigte. Es steht jedoch außer Zweifel, dass er als Kultusminister dauerhafte Erfolge erreicht hat: Mit seinem Namen sind - neben der Neuorganisation der Hochschul- und Sekundarbildung - die Einführung des achtjährigen Elementarunterrichts, die Lehrplanreform und die Entwicklung des institutionellen Systems der Volksbildung verbunden. ${ }^{14}$

Um die nationale Bildung auszuführen, betonte Hóman, dass er die Entwicklung von Schulen nachdrücklich unterstützen werde: „Die wichtigste Voraussetzung und auch die Grundlage der christlichen, nationalen Politik ist die Bestrebung für Modernisierung und Vertiefung der Volksbildung, die wir verfolgen müssen. Ich möchte die Arbeit von Eötvös und Wlassics, Apponyi und Klebelsberg ${ }^{15}$ fortsetzen. Meine Zielsetzung ist die Vertiefung der Volksbildung durch Bereitstellung materieller Voraussetzungen, Verbesserung von Bildung und Erziehung [...], die Entwicklung des christlich-ungarischen Geistes und der Lebensanschauung."16

Der Grund für den Schulbau war, dass es in Ungarn nicht genug Schulen gab, und diese wenigen Schulen waren auch nicht in einem guten Zustand, was dem Ideal der nationalen

\footnotetext{
${ }^{13}$ Siehe UJVÁRY, Hóman Bálint és népbírósági pere. ${ }^{14}$ Siehe HÓMAN, Magyar sors; HóMAN, Művelődéspolitika; MANN, Oktatáspolitikusok 79-126.

15 József Eötvös (7. 4. 1848-11. 9. 1848 und 20. 2. 18672. 2. 1871), Gyula Wlassics (15.1.1895-3.9.1903), Albert Apponyi (8. 4. 1906-17. 1. 1910), Kuno Klebelsberg (16. 6. 1921-24. 8. 1931) waren vorbildliche Kultusminister von Ungarn an der Wende des 19.-20. Jahrhunderts. Siehe BÖLÖNY, Magyarország kormányai.

${ }^{16}$ JÓBORÚ, Köznevelés 196-197.
} 
Bildung nicht entsprach. Der Bau von Schulen blieb ein zentrales Thema während der zweiten Amtszeit von Hóman, die Ausrichtung seiner Unterrichtspolitik wurde aber maßgeblich durch die Neuordnung der Bildung in den durch die Wiener Beschlüsse ${ }^{17}$ wiedergewonnenen Regionen bestimmt. In diesen Regionen (Siebenbürgen, Oberland - sog. Erdély, Felvidék) war es wichtig, sie in die Bildungsverwaltung des Mutterlandes zu integrieren und die Regulierung zu vereinheitlichen.

Nach der Rezession aufgrund der Wirtschaftskrise stellte Hóman größere Summen für den Schulbau bereit. Zwischen 1935-1940 wurden mehr als 2000 Volksschulen gegründet. ${ }^{18}$ Hóman dachte, dass die Überfüllung in Schulen und der schlechte Zustand von Schulgebäuden immer noch wichtige Probleme waren. Ein Vertreter stellte für den Kultusminister die Daten zusammen, damit er den Grad der Überfüllung prüfen konnte: Mehr als 1200 Lehrer unterrichteten in Klassen mit 70-80 Schülern, mehr als 500 Lehrer in Klassen mit 80-100, und mehr als 100 Lehrer in Klassen, in denen es 100-120 Kinder gab. Aufgrund der zur Verfügung stehenden landesweiten Daten in der Fachliteratur konnte man feststellen, dass bis 1940 von den geplanten 3000 Schulen nur 2200 gebaut wurden. ${ }^{19}$ Dies war jedoch im Verhältnis zur benötigten Anzahl und zur Bevölkerung des Landes bedeutungslos.

\section{Schulgründungen im Komitat Baranya ${ }^{20}$}

Die Dokumente der Schulaufsicht von Pécs und Baranya des MNL BAML berichten in dem Zeit-

\footnotetext{
${ }^{17} 2.11 .1938$ und 30. 8. 1940.

${ }^{18}$ MANN, Oktatáspolitikusok 95.

${ }^{19}$ JÓBORÚ, Köznevelés 195-196.

${ }^{20}$ Komitat Baranya liegt in Südtransdanubien, zu dieser Zeit an der jugoslawischen Grenze.
}

raum, der hier behandelt wird, darüber, dass viele Gemeinden um staatliche Beihilfen für Schulbau oder die Erhaltung ihrer Schulgebäude baten - mit mehr oder weniger Erfolg. Sie begründeten ihre Anträge zum Beispiel mit der Anzahl der schulpflichtigen Kinder vor Ort und mit Karten mit Angaben über den Wohnort der Schüler, detaillierten Kostenplänen bzw. mit Unterstützungserklärungen der lokalen kirchlichen oder weltlichen Behörden. In Ermangelung von Staatsbeihilfen suchten die Schulbehörden Kirchen, Gemeinden oder Vereine - mit finanziellen Schwierigkeiten nach einer anderen Lösung: Es war üblich, die staatliche Erhaltung der Schulen durch gemeindliche oder kirchliche Behörden zu initiieren. Neben diesen, von unten organisierten Bestrebungen findet man von oben, von der Ministerebene, organisierte Verstaatlichungen und Schulbauten. Hier werden Beispiele für alle diese Fälle genannt, aber es sollte hinzugefügt werden, dass es in den Archiven zahlreiche Fälle gab, von denen nur die typischsten für diesen Aufsatz ausgewählt wurden.

\section{Von unten initiierte staatliche Beihilfen bzw. Verstaatlichungen}

Es gab im Allgemeinen zwei Formen der staatlichen Unterstützung für den Schulbau, die von den gemeindlichen Behörden initiiert wurden: einerseits die nicht rückzahlungspflichtigen staatlichen Beihilfen und andererseits das komplementäre Darlehen.

Im August 1932 beantragte die römischkatholische Schule von Siklós beim Ministerium die Erlassung ihres Darlehens (8000 pengő). Der Abteilungsleiter des Ministeriums informierte die Gemeinde jedoch, dass er „,der Bitte der Kirchengemeinde grundsätzlich nicht nachkommen könne, jedoch gewähre er eine Stundung der Rückzahlung des Darlehens bis zum 1. Januar 1933, im Hinblick darauf, dass der Antrag begründet sei." Dies bedeutete nicht, dass die ganze Schuld ein halbes Jahr später fällig gewesen 
wäre, sondern dass die Pfarrei eine tilgungsfreie Zeit hatte, um die Rückzahlung bis dahin zu beginnen. In seinem Brief an die Schulaufsichtsbehörde fügte das Ministerium hinzu, warum das Darlehen nicht erlassen werden konnte: „Die Rückzahlung der vom Nationalen Volksschulbaufonds bewilligten Darlehen wurde bei der weiteren Durchführung des Volksschulbaus berücksichtigt und ist in jedem Fall unverzichtbar. Die Kirchengemeinde musste ihre finanziellen Möglichkeiten bei der Annahme der Rückzahlungsverpflichtung berücksichtigen, umso mehr, weil ich die römisch-katholische Kirchengemeinde von Siklós bei diesem Bau außerordentlich weitreichend unterstützt habe." ${ }^{21}$ Der letzte Punkt des Ministeriums ist gerechtfertigt: In den anderen untersuchten Fällen war die Höhe der staatlichen Beihilfen nicht so hoch wie in diesem Fall. Die Kirchengemeinde von Siklós bekam das Dreifache des Darlehens als staatliche Beihilfe (also 24000 pengő). Um die Maßnahmen ausführlicher darzustellen: Die römisch-katholische Kirchengemeinde von Beremend bekam zum Beispiel 5000 pengő als Beihilfe und 3000 pengő als Darlehen. ${ }^{22}$ Wenn man die Höhe der Beihilfen und das Darlehen bewerten möchte, darf man natürlich nicht vergessen, dass es sich um unterschiedlich große Gemeinden und eine andere Anzahl von schulpflichtigen Kindern handelt, in Anbetracht dessen kann man jedoch sagen, dass die staatliche Beihilfe für Siklós besonders hoch war.

Die römisch-katholische Kirchengemeinde in Vajszló bekam eine kleinere Menge für den Schulbau: 15000 pengő als staatliche Beihilfe und 6000 pengő als Darlehen; neben diesen Summen erhielt die Gemeinde 9000 pengő vom Ungarischen Katholischen Religionsfonds. Auch in diesem Fall gab es eine häufige Warnung, dass die Gemeinde, wenn sie mehr baue als

\footnotetext{
${ }^{21}$ MNL BaML VI. 502. 2042/1932.

${ }^{22}$ Ebd. 1149/1933.
}

bisher angenommen (z.B. eine größere Lehrerwohnung, mehr Klassenzimmer), die zusätzlichen Kosten selbst tragen müsste, da sie ursprünglich nicht im Antrag enthalten waren. In diesem Fall wissen wir auch mehr über den Nationalen Volksschulbaufonds: Das Darlehen musste zu einem Zinssatz von $6 \%$ an den Fonds zurückgezahlt werden. ${ }^{23}$ Aus diesem Fonds wurde also der kontinuierliche Schulbau finanziert wie schon vorher erwähnt, wurde aufgrund dessen die Schule von Siklós nicht von ihren Schulden befreit.

Das Verfahren zur Beantragung staatlicher Beihilfen lässt sich am Beispiel der Schule von Alsószentmárton nachvollziehen: Im Dezember 1938 reichte die römisch-katholische Kirchengemeinde von Alsószentmárton über die Verwaltungskommission des Komitats Baranya beim Kultusministerium einen Antrag auf Errichtung eines Klassenzimmers und einer Lehrerwohnung ein. Im April 1939 erhielt sie dafür 12000 pengő staatliche Beihilfe. Im November 1939 forderte der Hauptberater des staatlichen Architekturbüros den Minister auf, den Betrag der staatlichen Beihilfe von 12000 auf 16500 zu erhöhen; er fügte hinzu, dass der Gesamtbetrag 21000 pengő betragen würde, was sich um 1500 reduzieren ließe, wenn die Wände teilweise aus Lehm bestünden und die Kirchengemeinde nicht mehr als 3000 zum Schulbau beitragen könnte. Der Bau wäre jedoch sehr wichtig, da sich im Klassenzimmer 70 Kinder auf 50 Quadratmetern befänden und die Benutzung der Lehrerwohnung bereits die Erkrankung eines Familienmitglieds des Lehrers verursacht hätte. Ein Monat später, im Dezember 1939, gab die Kirchengemeinde ihren Plan auf, eine Schule zu bauen, weil sie die Kosten nicht decken konnte. Anschließend entschied sich das Ministerium für die ausnahmsweise Anhebung der staatlichen Beihilfen für den Bau

${ }^{23}$ Ebd. 2055/1932. 
um 6000 auf 18000 pengő wegen der schwierigen finanziellen Situation der Kirchengemeinde. ${ }^{24}$ Die Frage ist, warum sich der Minister schließlich dazu entschied, das Maß der staatlichen Beihilfen zu erhöhen, aber dies ermöglichte die Errichtung einer Schule in Alsószentmárton.

Neben der Menge der staatlichen Beihilfen und dem Verfahren zu deren Beantragung ist es wichtig, den Prozess der Gründung einer Schule selbst zu untersuchen, wenn eine Schule von unten, auf Initiative der lokalen Gemeinschaft gegründet wurde.

Die Organisationsarbeit dauerte mehrere Jahre in den Gemeinden Kölked, Debrina und Karapancsa in der Nähe von Mohács. Die Bildung der Kinder in der Region war aufgrund der größeren Entfernung zwischen den Höfen und den schwierigeren Straßenverhältnissen ein Problem. Der Antrag zur Errichtung der Schule wurde vom Lehrer József Gadányi zusammen mit einigen Einwohnern eingereicht. Sie begründeten ihren Antrag - neben der ungünstigen geographischen Lage der Gemeinden - mit nationalen Beweggründen. Sie sagten, dass die Errichtung der Schule in nationaler Hinsicht wichtig sei, weil diese Region nur von Ungarn bewohnt war. Angesichts der nach Trianon verbleibenden ethnischen Minderheiten war es besonders nötig, in ungarischer Sprache zu unterrichten und so viele ungarische Bildungseinrichtungen wie möglich einzurichten. Die gewaltsame Magyarisierung begann schon in der zweiten Hälfte des 19. Jahrhunderts, aber es kann festgestellt werden, dass sie im Einklang mit den Idealen der nationalen Bildung und der ungarischen kulturellen Überlegenheit immer noch als wesentliche Frage angesehen wurde. Nach dem Antrag wurden mehrere Behörden an dem Organisationsprozess beteiligt: Das Kultusministerium bat den Schulinspektor über die Verwaltungskommission, sich mit einem Mitglied des staatlichen

${ }^{24}$ Ebd. 481/1940.
Architekturbüros mit den Betroffenen über die Errichtung einer Schule zu beraten und den am besten geeigneten Ort für die Schule zu bestimmen. Dann erhielt der Schulinspektor von Baranya einen Brief des Hauptstuhlrichters des Bezirks Mohács, in dem der Hauptstuhlrichter ihn darum bat, über den Termin der lokalen Verhandlung informiert zu werden, da er zusammen mit dem Notar daran teilnehmen würde. Der Hauptstuhlrichter bemerkte, dass der Notar über die lokale Bevölkerung und die Bauernhöfe wertvolle Informationen geben könne. Der Schulinspektor des Komitats Bács-Bodrog nahm an dem Organisationsprozess teil, da es sich um ein Gebiet an der Grenze der Komitate handelte. Der Bürgermeister von Mohács bestätigte die Verbindung zwischen den Komitaten, indem er bekannt gab, dass die Mehrheit der schulpflichtigen Kinder der 44 Familien, die zur Mohács-sziget gehörten, in Hercegszántó, einer Gemeinde von Bács-Bodrog, zur Schule gingen. Der Notar übersandte auch einen Bericht über die Kinder der 57 Familien, die zu den anderen Gemeinden in der Nähe (Karapancsa, Debrina) gehörten, wo sie derzeit die Schule besuchten. Demzufolge könne festgestellt werden, dass diese Kinder in verschiedenen Siedlungen zur Schule gehen mussten, sodass es ratsam wäre, für sie eine gemeinsame Schule zu gründen. Die Dokumentation über die Errichtung der Schule ist bis zu dem Punkt verfügbar, wo das Ministerium eine abweisende Antwort gab: „Für die Ausgaben der Schule gibt es keine Deckung, somit kann ich diesen Wunsch nicht erfüllen".25 Dies war ein Antrag eines lokalen Lehrers und der Anwohner gewesen, eine öffentliche Schule $\mathrm{zu}$ errichten, sodass diese als eine der von unten initiierten Schulgründungen angesehen werden kann.

Im Interesse der nationalen Bildung kam die Idee der Verstaatlichung von konfessionellen

${ }^{25}$ Ebd. 502. 3005/1935. 
Schulen durch die gemeindlichen oder kirchlichen Behörden auf. Am 12. Juli 1939 schrieb der evangelische Geistliche an den Schulinspektor des Komitats Baranya hierzu Folgendes: „Während meiner Zeit bei Ihnen sprachen wir über den Wunsch, Volksschulen an der Grenze zu Jugoslawien im Süden von Baranya zu verstaatlichen, sowohl aus nationalen Gründen als auch aus Sicht der einheitlichen ungarischen Jugendbildung." Er drängte, dass die konfessionellen Schulen von Borjád und Magyarbóly nach Zustimmung der evangelischen und der römischkatholischen Kirche vom Staat übernommen werden sollten. Der Geistliche erläuterte die Umsetzung: Die Schule von Borjád mit 70 evangelischen und 30 römisch-katholischen Kindern in drei oder vier Klassenzimmern sollte verstaatlicht werden. Im Hinblick auf die religiöse $\mathrm{Zu}$ gehörigkeit der Kinder wurden zwei evangelische und ein römisch-katholischer Lehrer beschäftigt. Über Magyarbóly sprach der Geistlicher sehr wenig; er erwähnte nur, dass „wir die Schule dem Staat überlassen wollen“. ${ }^{26}$ Es gab mehrere Gründe dafür, dass die Kirche ihre Schulen dem Staat überlassen wollte. Entweder glaubten sie tatsächlich an das Ideal der nationalen Bildung und betrachteten die Verstaatlichung als das beste Instrument, wie der Geistliche schrieb, oder sie konnten die Schulen einfach nicht erhalten und waren gezwungen, sie $\mathrm{zu}$ verstaatlichen..$^{27}$

\section{Von der Ministerebene organisierte Verstaatlichung und Schulbau}

Im Bereich der von oben organisierten Verstaatlichungen tauchte die Frage der Kirche bzw. Gemeinden als Erhalter auf. 1932 schrieb der Abteilungsleiter des Ministeriums an den Schul-

\footnotetext{
${ }^{26}$ Ebd. 502. 1489/1938.

${ }^{27}$ Andere Verstaatlichungen von konfessionellen und gemeindlichen Schulen im Komitat Baranya an der Wende des 19.-20. Jahrhunderts siehe in NiKLAI, Die Modernisierung.
}

inspektor von Baranya über die schulpflichtigen Kinder von Kárászpuszta. Darin erinnerte er den Schulinspektor daran, dass "der Unterricht in Kárászpuszta seit dem 1. September 1930 pausiert und 32 Schüler ohne Bildung sind." Er fügte auch Folgendes hinzu: „Die Konfessionen sind nur berechtigte Schulerhalter; wenn sie dieses Recht nicht ausüben wollen, dann tritt gemäß § 23. der GA 1868:XXXVIII die Verpflichtung der politischen Gemeinden in Kraft. Es steht jedoch außer Zweifel, dass nicht nur die Interessen der religiös-moralischen Erziehung, sondern auch finanzielle Erwägungen den weiteren Bestand der bewährten konfessionellen Schulen garantieren." Anschließend forderte er den Schulinspektor dazu auf, einen Kompromiss zwischen der lokalen Kirchengemeinde und der „politischen“ (weltlichen) Gemeinde zu erreichen, und er sollte darauf hinweisen, dass es für die Gemeinde immer noch vorteilhafter sei, konfessionelle Schulerhalter neben „pädagogischen Erwägungen" auch finanziell stärker zu unterstützen, als die Schule alleine zu erhalten. Er fügte hinzu, dass der Schulinspektor, falls dieser Kompromissversuch erfolglos wäre, die Verwaltungskommission kontaktieren sollte, um „die politische Gemeinde zu verpflichten, eine Schule mit der gleichen Größe wie die Zahl der Schüler zu errichten." Im Januar 1933 kam die Anfrage des Ministeriums über das Ergebnis der Einschreibung von schulpflichtigen Kindern an. Am 20. April 1933 schickte der Notar von Dencsháza (Kárászpuszta gehörte verwaltungsrechtlich zu Dencsháza) dem Schulinspektor die Dokumente, die für die Organisation der Schule angefordert worden waren, und verband den Fall von Kárászpuszta mit dem Ideal der nationalen Bildung: „Schließlich möchte ich Sie sehr bitten, dass der Fall der Schule von Kárászpuszta, der schon seit vier Jahren anhängig ist, schnell gelöst wird, denn die traurige Tatsache ist, dass 50-60 Kinder jetzt schon im vierten Jahr ungebildet sind, und niemand kann das gleichgültig mitansehen, wenn es um die Erziehung 
von ungarischen Kindern geht." Eng verbunden mit unserem Thema über das abenteuerliche Schicksal der Schule ist nur die Tatsache, dass das alte Schulgebäude schließlich vom Nationalen Landeskreditinstitut für Kleinbauern zurück gemietet wurde und die Gemeinde von Dencsháza der Schulerhalter wurde. Wahrscheinlich wurde mit den Konfessionen kein Kompromiss gefunden, oder dies war das Ergebnis des Kompromisses, das kann anhand der Dokumente nicht genau geprüft werden. ${ }^{28}$

Im Januar 1942 begann eine großflächige, auf Ministerebene organisierte Schulbau-Aktion; in Baranya wurden mehr als 40 Schulen eingerichtet. Der Oberstudiendirektor informierte im März den Schulinspektor über die Gemeinden, in denen der Staat eine Schule plante, und darüber, wie viele Lehrerstellen eingerichtet werden sollten. In der zweiten Tabelle finden sich diese Daten. Die meisten Schulen wurden mit ein bis zwei Lehrern organisiert, für kleinere Siedlungen, die den dringendsten Bedarf an Schulen hatten. In mehreren Dörfern wurde, wie bereits erwähnt, die Bedeutung der ungarischen Sprache stärker, und man versuchte, dies nach Trianon im Einklang mit dem Ideal der nationalen Bildung $\mathrm{zu}$ betonen. Interessant ist, dass man in Bellye nicht nur die Einrichtung der ungarischen Sektion einforderte, sondern auch die sofortige Umlenkung der ungarischsprachigen Kinder von der deutschen Sektion. ${ }^{29}$

\section{Zusammenfassung}

Im Kern der ungarischen Kulturpolitik stand im untersuchten Zeitraum die christlich-nationale Ideologie und die ungarische kulturelle Überlegenheit; diese bildeten die Grundlage der Volksunterrichtspolitik von Bálint Hóman. Die Schulgründungen basierten auf den Schlüsselwörtern der Ära: patriotische, nationale, religiösmoralische Erziehung. Die zentrale Unterrichtspolitik war aber etwas ehrgeiziger als ihre Umsetzung, was anhand lokaler Beispiele geprüft wurde. Es gab sowohl von unten (Gemeinden und andere Schulerhalter) organisierte Bestrebungen, als auch von oben, von der Ministerebene organisierte Verstaatlichung und Schulbau. Die Rolle des Staates war eine immer wiederkehrende Frage in der Geschichte der Schulen seit dem 19. Jahrhundert. Ab Anfang der 1930er Jahre gibt es Beispiele für Verstaatlichungen, bei denen die staatliche Übernahme dazu führte, dass der konfessionelle Charakter einer Schule verloren war, weil sie keinen angemessenen Schulbesuch ermöglichte. Im Bereich der staatlichen Schulgründungen wurden 1942 - in Bezug auf das Komitat Baranya - gewaltige Arbeiten angefangen und mehr als 40 Einrichtungen von staatlichen Schulen vorbereitet.

\footnotetext{
${ }^{28}$ MNL BaML VI. 502. 1152/1940.

${ }^{29}$ Ebd. 502. 939-1088/1942.
} 


\section{Tabellen}

A tanulók vallósi megoszlása az iskolák jellege szerint

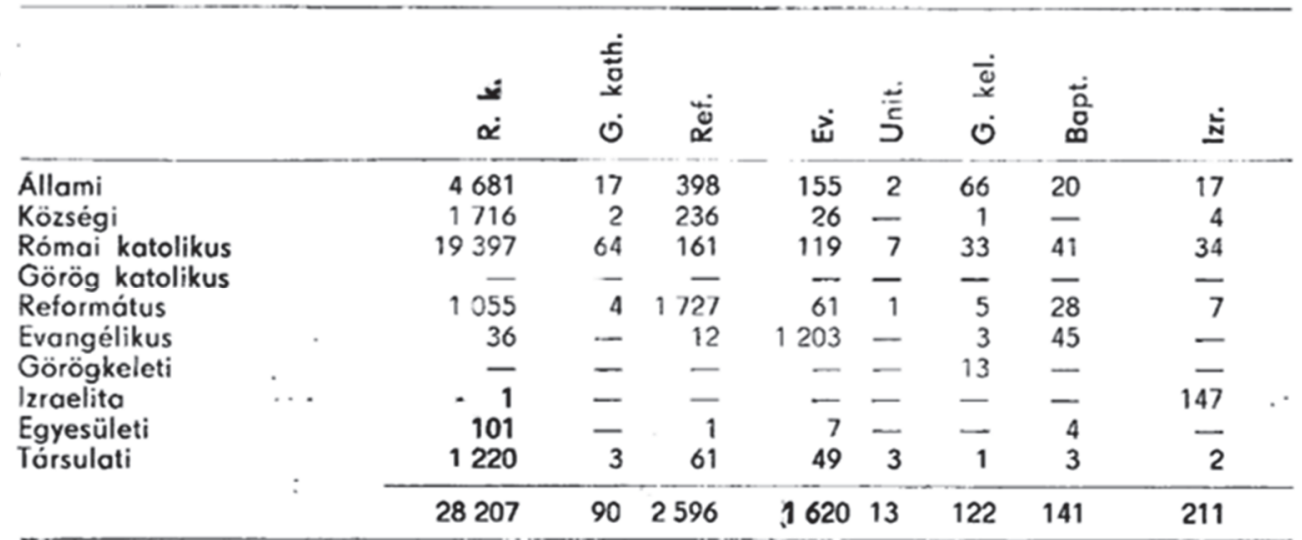

Tabelle 1. „Konfessionelle Verteilung der Schüler nach Erhalter der Schulen” in Komitat Baranya

Senkrechte - Erhalter: Staat, Gemeinde, römisch-katholische, griechisch-katholische, reformierte, evangelische, griechisch-orthodoxe, israelitische Konfession, Verein, Sozietät

Waagerechte - Konfessionen: römisch-katholische, griechisch-katholische, reformierte, evangelische, unitarische, griechisch-orthodoxe, baptistische, israelitische Konfession

(LAKI, A népiskolák fejlődése 220.) 


\begin{tabular}{|c|c|c|}
\hline Gemeinden, wo Schule eingerichtet wurde & Lehrer & Weitere Bemerkung \\
\hline $\begin{array}{c}\text { Petárda-Zrínyi vitézi telep, Benge, } \\
\text { Jenőfalva, Kö, Dárda-Uglyespuszta, } \\
\text { Kisdárda, Baranyavár-Cukorgyárnál, } \\
\text { Bolmány-Ujbolmány, Bolmány-Horthy M. } \\
\text { vitéz telep }\end{array}$ & 1 & \\
\hline $\begin{array}{c}\text { Várdaróc, Újbezdán, Keskend, Izsép, } \\
\text { Laskó-Keskenyerdő, Kopács, Sepse, } \\
\text { Nagybodolya, Bolmány, } \\
\text { Baranyaszentistván, Benge-Braidaföld } \\
\text { puszta }\end{array}$ & 2 & \\
\hline Torjánc, Hercegmárok & 2 & $\begin{array}{l}\text { „Einrichtung einer ungarischen } \\
\text { Sektion für ungarische Kinder" }\end{array}$ \\
\hline Darázs, Laskó, Hercegszöllős, Csuza, Lőcs & 3 & \\
\hline Petárda, Baranyavár, Albertfalu, Dályok & 3 & $\begin{array}{l}\text { "Einrichtung einer ungarischen } \\
\text { Sektion für ungarische Kinder" }\end{array}$ \\
\hline Bellye & 3 & $\begin{array}{c}\text { „3 ungarisch sprachige Kinder } \\
\text { besuchen die deutsche Sektion. } \\
\text { Sofort in die ungarische Sektion } \\
\text { umleiten." }\end{array}$ \\
\hline $\begin{array}{l}\text { Vörösmart, Hercegszöllös-Frigyesföld, } \\
\text { Karancs }\end{array}$ & 4 & \\
\hline Baranyabán & 5 & \\
\hline Laskafalu & 5 & $\begin{array}{l}\text { "Einrichtung einer ungarischen } \\
\text { Sektion für ungarische Kinder" }\end{array}$ \\
\hline Kiskőszeg, Dárda & 6 & \\
\hline Kázsfalu & 6 & $\begin{array}{l}\text { "Einrichtung einer ungarischen } \\
\text { Sektion für ungarische Kinder" }\end{array}$ \\
\hline Pélmonostor & 7 & $\begin{array}{l}\text { "Einrichtung einer ungarischen } \\
\text { Sektion für ungarische Kinder" }\end{array}$ \\
\hline
\end{tabular}

Tabelle 2. Dateien über den von Ministerebene organisierenden Schulbauen im Komitat Baranya im Jahr 1942

(Sammeln der Verfasserin nach MNL BaML VI. 502. 939 - 1088/1942) 


\section{Korrespondenz:}

Dr. Patrícia Dominika NIKLAI

Universität Pécs

Staats- und Rechtswissenschaftliche Fakultät

Dezső-Márkus-Forschungsgruppe für Vergleichende Rechtsgeschichte

48-as tér 1

$\mathrm{H}-7622$ Pécs

niklai.dominika@ajk.pte.hu

ORCID-Nr: 0000-0003-4532-3199

\section{Abkürzungen:}

MNL BaML = Komitatsarchiv Baranya des Ungarischen Landesarchivs VI. 502. Bvm. és Pécs v. Tanfelügyelőségének iratai

Siehe auch das allgemeine Abkürzungsverzeichnis: [http://www.rechtsgeschichte.at/media/abk.pdf]

\section{Literatur:}

ANONYMUS, A kultúrfölény és a jellemnevelés, in: Néptanítók Lapja 67/2 (15. 1. 1934) 41-42.

J. BÖLÖNY, Magyarország kormányai 1848-1975 (Budapest 1978).
Gy. Drozdy, Gróf Teleky Pál - Hóman Bálint, in: Néptanítók Lapja 72/5 (1.3. 1939) 149-151.

E. Cs. Herger, Auf dem Weg zur Autokratie? Kirchenpolitik in der Horthy-Ära in Ungarn. Krakowskie Studia z Historii Panstwa i Prawa 10 (2017) 69-95.

B. HómAN, Magyar sors - magyar hivatás (Budapest 1942).

DERS., Művelődéspolitika (Budapest 1938).

M. JóBORÚ, Köznevelés a Horthy-korszakban (Budapest 1972).

J. LAKI, A népiskolák fejlődése, illetve stagnálása a két világháború közötti Baranyában, in: J. SzITA (Hg.), Baranyai helytörténetírás (Pécs 1983) 213-254.

M. MANN, Oktatáspolitikusok és koncepciók a két világháború között (Budapest 1997).

P. T. NAGY, A növekvő állam árnyékában. Oktatás, politika 1867-1945 (Budapest 2011).

P. D. NIKLAI, Die Modernisierung des Volksunterrichts in Ungarn. Umsetzung des Gesetzes über den Volksunterricht im Komitat Baranya nach 1868, in: Journal on European History of Law 2 (2018) 231-235.

Országgyűlési Almanach az 1939-44. évi Országgyülésről (Budapest 1940). G. UjvÁRY (Hg.), Hóman Bálint és népbírósági pere (Budapest 2019).

G. UJvÁRY (Hg.), Történeti átértékelés. Hóman Bálint, a történész és a politikus (Budapest 2011). 